\title{
Detonation Propagation in Stratified Reactant Layers
}

\author{
Gaathaug A.V. Vaagsaether K. Lundberg J. Bjerketvedt D. \\ Department of Process, Energy and Environmental Technology, University College of Southeast Norway, Norway, \\ andre.v.gaathaug@usn.no
}

\begin{abstract}
A numerical investigation of detonation propagation in stratified reactant layers is presented in this paper. It is baesed on the reactive Euler equations using a two step chemical kinetics approach. The numerical simulations are based on the reactive Euler equations. Turbulence is solved with a one equation model, and the chemical kinetics is modeled as a two steps. The first step is an induction time step, and the second step is an exothermic step. The numerical setup is scaled to keep the numerical resolution of the induction zone constant to 10 cells. Initial simulations were conducted to generate detonation structures in homogeneous reactants and with cyclic boundary conditions. The developed structures were mapped into a domain with a stratified reactant layer on top of a inert layer. The results show that the detonations fail to propagate as the triple points of the propagating detonation is "lost" into the inert layer.

Keywords: CFD, Detonation, Simplified kinetics
\end{abstract}

\section{Introduction}

Denotations are supersonic combustion waves relative to the reactants in front of the wave. Its subsonic counterpart is known as deflagrations. The two modes of combustion differ fundamentally as the deflagration is driven by mass diffusion into the reaction zone and heat conduction from the reaction zone into the reactants. The downstream boundary conditions are also important, as the combustion product density is lower than the reactants. The detonation propagates as a leading shock wave in front of the reaction zone and compresses the reactants and increasing the temperature. This increased temperature and pressure lead to an onset of exothermic chemical reactions.

The 1D CJ (Chapman-Jouget) theory gives the detonation velocity $S_{C J}$ as a function of the reactant initial conditions and the energy released in the reaction zone, hence it is a property of the reactant mixture, (Lee, 2008). In the $\mathrm{CJ}$ theory, the detonation is evaluated at the upstream and equilibrium downstream conditions.

The extension of the Chapman-Jouget theory is known as the ZND (Zel'dovich (Zel'dovich, 1940), Neumann (von Neumann, 1963) and Döring (Döring, 1943)) theory, which is a $1 \mathrm{D}$ consideration of detonations. In the ZND theory, a leading shock (no reactions) is followed by a induction zone, where firstly radicals are produced and later follows the exothermic reaction zone where heat is pro-

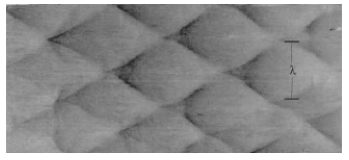

Figure 1. Results from detonation experiments. Sooted plate triple point trajectories of structured cellular pattern. From (Austin and Shepherd, 2003)

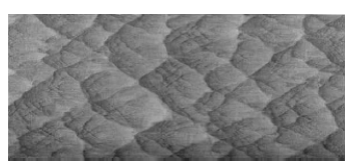

Figure 2. Results from detonation experiments. Sooted plate triple point trajectories of unstructured cellular pattern. From (Austin and Shepherd, 2003)

duced. An illustration of the pressure across a detonation wave is given in figure 3 . The state behind the leading shock wave is referred to as the von Neumann state (example velocity: $u_{v N}$ ). It is given by the normal shock relations, and the state in front of the wave.

Real detonations are three dimensional and highly unsteady. They are driven by shock-shock interactions, and a dominant feature of real detonations are the trajectories of the shock triple point. Depending on the reactant mixture, the trajectories could be regular or irregular when recorded on sooted plates inside experimental equipment. Two examples are given in figures 1 and 2, from (Austin and Shepherd, 2003).

Fluid dynamic simulations of detonations must cap-

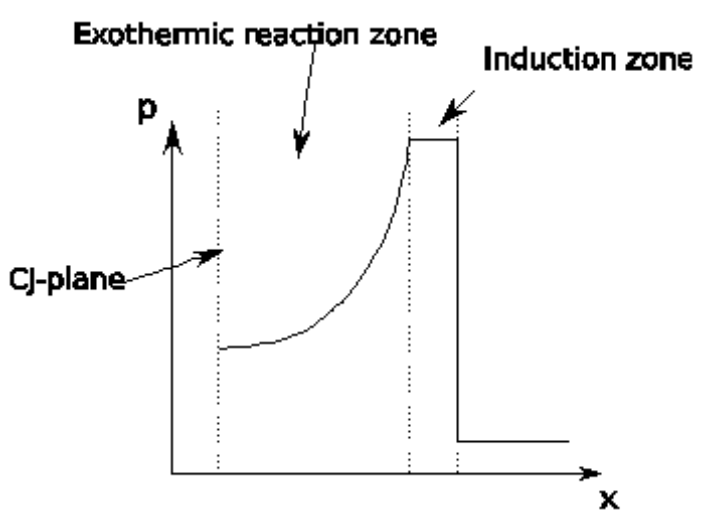

Figure 3. Pressure profile across a detonation front as described by the ZND theory. 
ture the important physics of the phenomenon as well as the chemistry. As real gaseous detonations involve real gaseous reactions. The most simple reactant system is the $\mathrm{H} 2-\mathrm{O} 2$, which can include 8 species and up to 21 elementary reactions. It is manageable to include them in simulations, but more complex fuel-air mixtures can reach many hundred species and thousands of reactions.

Detonation propagation in stratified fuel layers, also known as semi confined detonations, has gained a recent interest in the safety research community as well as the jet propulsion research community. One aspect is related to the emerging world wide hydrogen infrastructure where the safety aspect must be clarified. An other classical aspect of hydrogen safety is the nuclear industry, where hydrogen leakage has been a valid concern since the first reactors were built. An accidental leak of hydrogen will likely form a fuel layer at the ceiling, where it is bound by a solid ceiling above and an compressible boundary of inert air below. The same issue apply to dense gas leakage which could make a combustible layer of reactants along the floor.

In jet propulsion research there is an effort to make a rotating detonation engine. This engine will inject reactants axially and have a tangentially propagating detonation propagating through the reactants. In the system the detonation will be bound by a solid inlet wall on one side and combustion products on the other side.

A sketch of the problem is given in figure 4 . The shock plane is the leading shock wave, as no transverse waves are included. The CJ plane represent the end of the exothermic reactions. The shock is an oblique wave into the inert, while the dotted line is the contact surface. The expansion into the inert is drawn as a fan at the CJ plane. This is of course a simplified illustration of the problem.

This work aim to understand detonation propagation in reactant layers, especially the limits to where the detonation will fail. Recent studies by the KIT group and the Warsaw University of Technology (Grune et al., 2011; Rudy et al., 2013) have found a criteria for successful detonation propagation in a layer to be three cell size thick. An other study show that the critical layer thickness varies with the mixtures (Grune et al., 2016). The Warsaw University of Technology group showed that addition of methane to a hydrogen-air mixture increased the critical layer thickness, as shown by Rudy et al. (Rudy et al., 2016). A study by Houim and Fievisohn (Houim and Fievisohn, 2017) investigated the influence of the ratio of acoustic impedance between the reactant and inert layer on the detonation propagation. Their work focused concluded that a much denser inert or a much lighter inert gas is required to have a successful detonation propagation. They showed numerically how new triple points originate from the interface between inert and reactants. The earlier work by Sommers (Sommers, 1961) also showed the influence on inert gas density on the propagation of detonations. It was discussed how the diffraction gave a lower pressure and temperature, and also how a higher adiabatic index gave a higher post shock pressure and thus a lower velocity deficit. The influence of adiabatic index is not discussed in this work.

The current study aims to address the detonation propagation problem based on a simplified analysis, described later. The overall procedure includes initial simulations to establish several credible detonation structures. The credibility criteria are explained later. And the detonation structures are later mapped onto a domain with reactants on top of an inert gas layer. This paper will mostly focus on the simulation method, as the actual results are believed to be more interesting for a specialized audience.

\section{Numerical method}

The numerical method is based on the 2D Euler equations, where mass momentum and energy is solved using a flux limited centered scheme. It is developed in MATLAB and details on the TeleCoDet code is given by Vaagsaether (Vaagsaether, 2010).

$$
\begin{array}{r}
\frac{\partial \rho}{\partial t}+\frac{\partial}{\partial x_{i}}\left(\rho u_{i}\right)=0 \\
\frac{\partial \rho u_{i}}{\partial t}+\frac{\partial}{\partial x_{j}}\left(\rho u_{j} u_{i}\right)=-\frac{\partial p}{\partial x_{i}} \\
\frac{\partial E}{\partial t}+\frac{\partial}{\partial x_{i}}\left(u_{i} E\right)=-\frac{\partial}{\partial x_{i}}\left(p u_{i}\right)
\end{array}
$$

The energy $\mathrm{E}$ is given by the internal energy (given by the pressure and heat capacity ratio $\gamma=\frac{C_{p}}{C_{v}}$, kinetic energy and change of enthalpy energy due to chemical reactions:

$$
E=\frac{p}{\gamma-1}+\frac{1}{2} \rho u_{i} u_{i}+E_{c h}
$$

The turbulence is modeled as transport of turbulent kinetic energy with source and sink terms. More details of the numerical method is given by Vaagsaether (Vaagsaether, 2010).

The exothermic chemical reactions of a detonation is given as a source in the energy equation. It is modeled in two steps as a simplification of a real combustion wave. Both steps are modeled as transported progress variables. The $\alpha$ variable is the normalized induction time progress variable. $\alpha$ varies between 0 and 1 , where $\alpha=0$ represent a gas mixture too cold to start exothermic chemical reactions. A $\alpha=1$ represent a gas where the exothermic reactions can start. The $\beta$ is the normalized exothermic reaction progress variable, where $\beta=0$ is reactants and $\beta=1$ is products.

$$
\begin{aligned}
& \frac{\partial \rho \beta}{\partial t}+\frac{\partial}{\partial x_{i}}\left(\rho u_{i} \beta\right)=\dot{\omega} \\
& \frac{\partial \rho \alpha}{\partial t}+\frac{\partial}{\partial x_{i}}\left(\rho u_{i} \alpha\right)=\dot{\vartheta}
\end{aligned}
$$




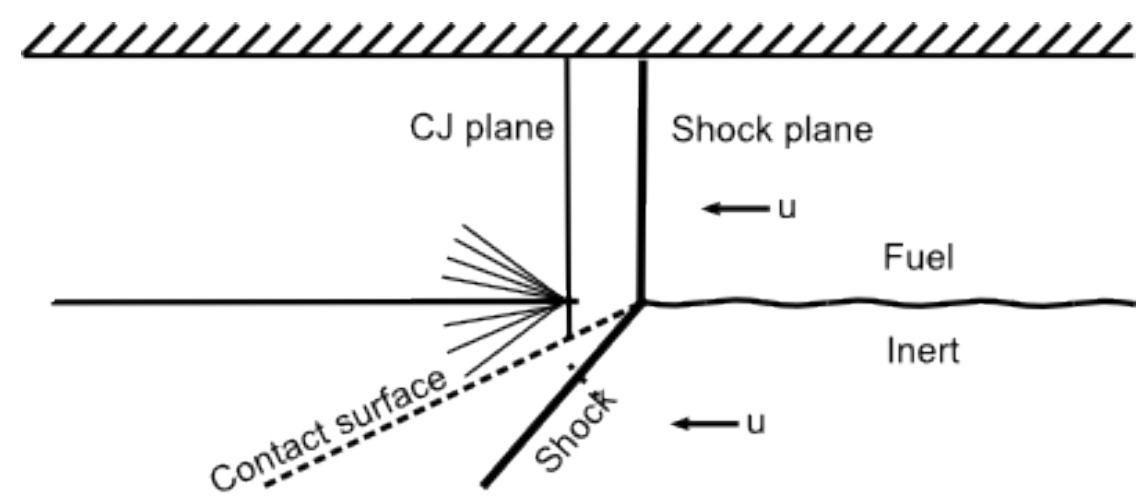

Figure 4. A sketch of a simplified wave structure where a detonation propagate in a stratified reactant (fuel) layer.

The idea behind this method is to constrain the $\beta$ source term, i.e. the heat produced by exothermic reactions, until after an induction period (modeled by the $\alpha$ variable). The total $\beta$ source term is given by:

$$
\dot{\omega}=\max \left[\dot{\omega}_{t}, \dot{\omega}_{k}\right]
$$

Where the $\dot{\omega}_{t}$ is the reaction rate given by the gradient of $\beta$. It is also given by the unburned gas density and the turbulent burning velocity $S_{T}$. The gradient of $\beta$ term is used to model the combustion propagation of deflagrations. The turbulent burning velocity is modeled as given by Flohr and Pitsch (Flohr and Pitsch, 2000).

$$
\dot{\omega}_{t}=\rho_{u} S_{T} \sqrt{\sum_{i}\left(\frac{\partial \beta}{\partial x_{i}}\right)^{2}}
$$

The kinetic term of the $\beta$ source is given by an Arrhenius type kinetics.

$$
\dot{\omega}_{k}= \begin{cases}0 & \alpha<1 \\ B(1-\beta) \exp \left(-\frac{E_{\beta}}{R T}\right) & \alpha=1\end{cases}
$$

The $E_{c h}$ of the energy equation is given by the progress variable and the change of enthalpy per unit mass of reactants $(q)$ as

$$
E_{c h}=\rho q(1-\beta)
$$

The induction time progress variable $(\alpha)$ source term is model with an Arrhenius kinetics term as

$$
\dot{\vartheta}=\rho A \exp \left(-\frac{E_{\alpha}}{R T}\right)
$$

Numerical simulations are sensitive to the numerical resolution, especially reacting flow with a high temperature dependence. A key concept in this study is to limit

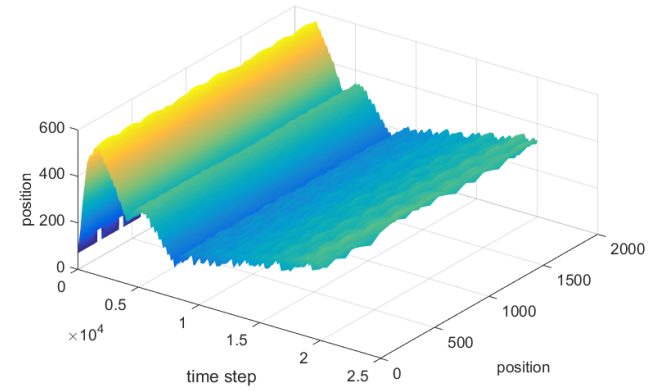

Figure 5. The detonation front for all time steps. Variables are: $q=30$ and $E_{\alpha}=30$. This is expected to give regular cellular structure of the front.

the variables of investigation to the energy $(q)$ and the induction time activation energy $\left(E_{\alpha}\right)$. All other variables should be constant. To keep the numerical resolution constant, the pre-exponential coefficient $A$, was adjusted to give 10 cells in the induction zone. This was calculated for a stable 1D detonation as, the simulation cell size $\Delta x=0.1$ :

$$
\left(u_{v N}-S_{C J}\right) \tau=\left(S_{C J}-u_{v N}\right) A \exp \left(\frac{E_{\alpha}}{R T}\right)=1
$$

which gives the pre-exponential factor to be

$$
A=\frac{1}{\left(S_{C J}-u_{v N}\right) \exp \left(\frac{E_{\alpha}}{R T}\right)}
$$

For the numerical setup, the initial pressure and density were both set to 1 , as well as the gas constant. The heat capacity ratio $\gamma$ was set to 1.4 and the input to the $\beta$ progress variable source term was: $B=4.9738$ and $E_{\beta}=15$.

\section{Initial calculations}

\subsection{Setup}

The detonations were simulated two dimensionally, as a simplification of the real three dimensional structure of 
detonation front. The first step of calculation was to develop a realistic $2 \mathrm{D}$ detonation structure. It was initiated as a slightly perturbed front with a 10 cells induction zone, and a 50 cells reaction zone. The top and bottom boundary condition (BC) were cyclic to account for an infinitely large domain. The right hand side inlet $\mathrm{BC}$ was zerogradient with a semi-constant velocity (velocity was adjusted during the simulation). The left hand side outlet was a zero-gradient BC.

\subsection{Results}

This setup had to run until it developed wave structure with transverse waves propagating up and down the leading shock of the detonation. This is also the structure of real detonations. In real detonations the transverse waves propagate in two perpendicular directions to the leading shock. The point of collision of these three waves is known as the triple point, and will leave a cellular pattern (see figure 1 and 2) on sooted surfaces in experimental investigations of detonations. In the $2 \mathrm{D}$ simulations a pattern will be made of the leading shock and the updown transverse shock. To investigate this, the pressure was used to find the position of the leading shock in the domain. This leading shock position was averaged for every time step to give a representation of the curvature (i.e. semi-triple-point recording). An illustration of the detonation front is shown in figure 5.

To investigate if the detonation simulations was developed, two parameters were used. One was the overall induction zone length, which was supposed to be close to 10 cells. This varied in the beginning of the simulation, but converged to 10 . The second parameter was the development of cellular structure of the detonation front. For this, an analysis was developed to find the cellular pattern in the detonation front history. It was based on gradient evaluation and thresholding, as well as artificial diffusion. At last it was converted to a binary variable and analyzed with a tool used for image analysis. The result is shown in figure 6. It is shown that the cells appear some time after initiation, and that the development of cell sizes take some time before they converge.

When the numerical variables were changed, the cellular pattern also changed. It is expected from the theory by Lee and Stewart (Lee and Stewart, 1990), that a more irregular cellular pattern would be the result of increased activation energy of the $\alpha$ variable. This can bee seen in figure 7. It is also shown that the variance of cell sizes is increased. The cyclic boundaries of the simulation was assumed to not influence the cell size, as long as there were more than three cells in the total height of the domain.

\section{Simulations of detonations in strati- fied reactant layers}

A 2D approach was used to investigate the detonation propagation in stratified reactant layers bound by one solid wall and an inert.

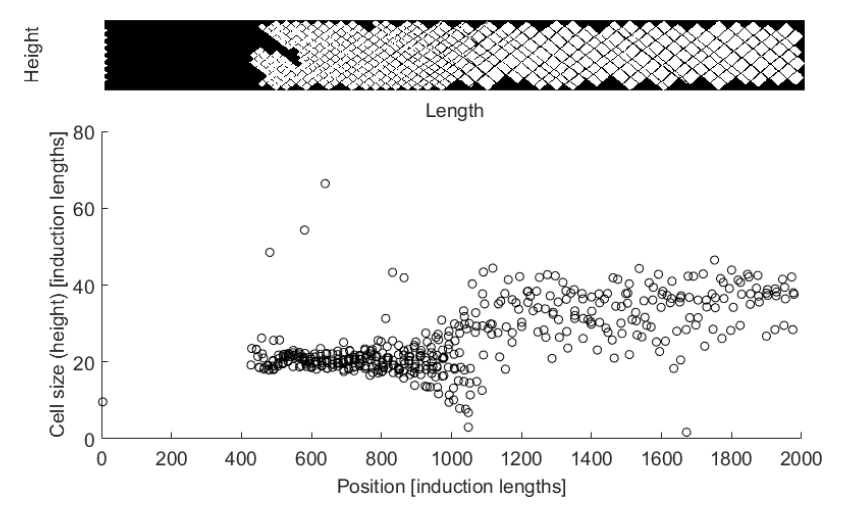

Figure 6. Top: Cellular pattern from the detonation front history. Bottom: The size of the cells vs the position. Variables are: $q=30$ and $E_{\alpha}=30$. This is expected to give regular cellular structure of the front.

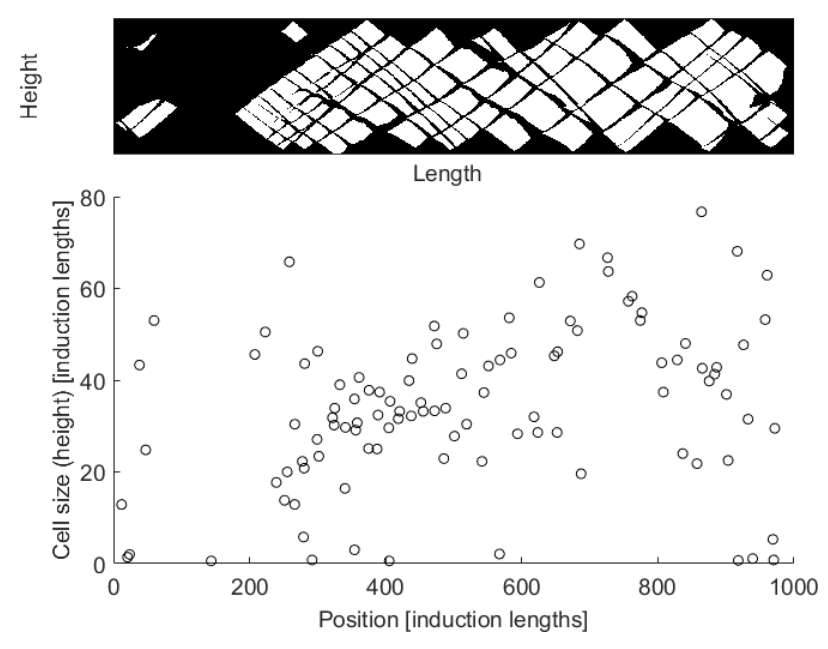

Figure 7. Top: Cellular pattern from the detonation front history. Bottom: The size of the cells vs the position. Variables are: $q=30$ and $E_{\alpha}=50$. This is expected to give an irregular cellular structure of the front. 


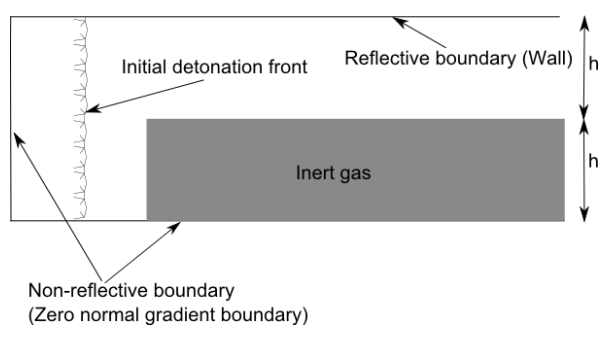

Figure 8. A sketch showing how the computational domain is set up with the mapped detonation structure to the left, the solid wall at the top and the inert layer along the lower boundary.

\subsection{Setup}

The initial simulations were mapped onto a larger domain where the inert was mapped as well. A sketch of the computational domain is given in figure 8 .

The right, left and bottom $\mathrm{BC}$ was a zero gradient, while the top wall was a reflecting $\mathrm{BC}$. The domain was moving to enable an investigation of the cellular structure by integrating (over time) the pressure in the induction zone. This makes up a more correct cellular structure as the thickness of the integrated variable represent the thickness of the induction zone. As this zone thickens, it is also expected that the detonation will fail and change to a deflagration following a leading shock wave.

\subsection{Results and discussion}

The detonation propagation is illustrated in figure $9\left(E_{\alpha}=\right.$ 30) where the triple point trajectories are shown and also how they are "lost" into the inert below the reactants. This is assumed to be the main reason for the failure of the detonation, as it will lead to an expansion loss into the inert. This gives a velocity deficit of the detonation, which result in a reduced strength of the leading shock wave, hence a lower von Neumann temperature and a longer induction zone. This eventually cause the detonation to fail after 1800 induction lengths.

The simulation results in figure 9 show that despite fulfilling the three detonation cells in the layer thickness this detonation still fails to propagate in the layer. A comparable real mixture to the case shown i figure 9 is stoichiometric hydrogen-air mixtures. As a comparison, that mixture have expected cell sizes of $8 \mathrm{~mm}$. The failure length would then be about $80 \mathrm{~mm}$ to $100 \mathrm{~mm}$. The regularity of the detonation cellular pattern is also connected to the propensity to generate new triple points. The main reason for this is the increased activation energy. With a higher activation energy the reaction becomes more sensitive to small perturbations, and could make new triple point on its own. By keeping the layer thickness fixed to 150 induction lengths, an irregular cellular pattern case was investigated, see figure 10. This was expected to be able to propagate longer into the reactant layer compared to the $E_{\alpha}=30$ case. It is clearly not the case, as one possible cause to the issue is the reduced number of detonation cells in the layer thickness. Much of a problem is also the definition of a cell
Table 1. List of all simulations in the project.

\begin{tabular}{cll}
\hline Layer thickness [ind length] & $\mathrm{q}$ & $E_{\alpha}$ \\
\hline 100 & 25 & 30 \\
100 & 30 & 30 \\
100 & 30 & 40 \\
100 & 30 & 50 \\
100 & 30 & 60 \\
150 & 25 & 30 \\
150 & 30 & 30 \\
150 & 30 & 40 \\
150 & 30 & 50 \\
\hline
\end{tabular}

size, as it is very much an expected size with a rather large variance.

The project has so far investigated 9 cases, each take about 1 to 2 weeks to simulate on a standard workstation, using about $30 \mathrm{~GB}$ memory. It typically has a $9 e 6 \mathrm{nu}-$ merical cells, solve 24 equations in each cell up to 15000 times. The simulation has to be adjusted by stepwise skipping the domain to the right as the simulation progresses. The list of completed cases is given in table 1 . The results so far show that all detonations fail at a short distance after entering the stratified reactant layer.

A further work on this project is first to simulate cases with a higher enthalpy per unit mass of reactants to investigate if it is within the limit of successful detonation propagation. The other case will be to further thicken the reactant layer, and lastly include a denser gas as the inert to allow for wave reflections at the boundary.

\section{Summary}

This paper have reported some of the work done on simulating detonations in stratified reactant layers. The simulations are based on the reactive Euler equations with a two step model for the chemical kinetics. The first step is a waiting time (induction time) progress variable which represent the induction time seen in simulations of detailed chemical kinetics. the second step is the exothermic reaction progress variable, which is linked to the energy equation. The numerical setup varied the change of enthalpy due to chemical reactions $q$ and the induction time activation energy $E_{\alpha}$. The numerical resolution was set to 10 cells in the induction zone (calculated on 1D steady state). The initial simulations had to run for a long time to ensure a developed detonation front. Due to the unsteady nature of detonations, a 10 cells criteria was used as well as a steady cellular detonation front. The initial detonation structure was mapped onto a stratified domain with proper boundary conditions and the failure on detonation was observed. The triple points were lost into the inert layer, and the expansion into the inert lead to a velocity deficit. This was assumed to be the reason for the detonation failure. The expected criteria from the literature of three detonation cells in the height of the reactant layer, 


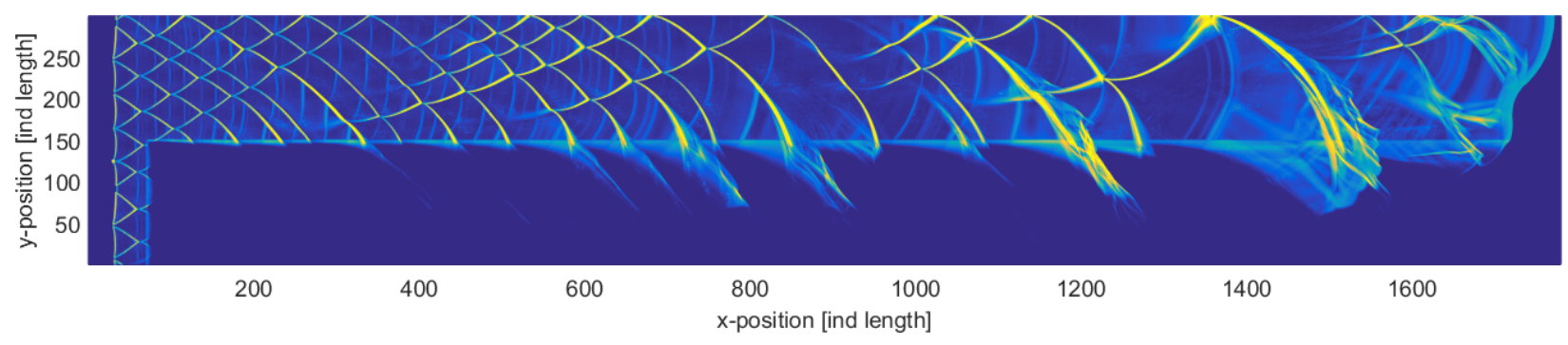

Figure 9. The triple point trajectory of a detonation in a stratified reactant layer. Variables are: $q=30$ and $E_{\alpha}=30$.

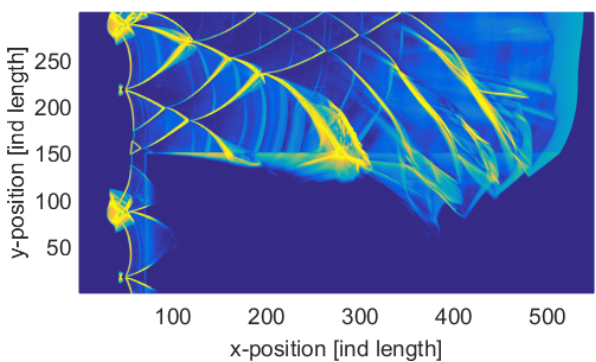

Figure 10. The triple point trajectory of a detonation in a stratified reactant layer. Variables are: $q=30$ and $E_{\alpha}=50$.

did not seem to be a general criteria for successful detonation propagation. The detonations with more irregular detonation cellular patterns also had a large variance i cell sizes, thus it might not be valid to base the thickness on the expected cell size. All simulated cases showed that the detonation failed as it propagated in the stratified reactant layer.

\section{References}

J.M. Austin and J.E. Shepherd. Detonations in hydrocarbon fuel blends. Combustion and Flame, 132(1-2):73-90, 2003. ISSN 00102180. doi:10.1016/S0010-2180(02)004224. URL http://linkinghub.elsevier.com/ retrieve/pii/s0010218002004224.

W. Döring. Über Detonationsvorgang in Gasen [On detonation processes in gases]. Annals of Physics, 1943.

P. Flohr and H. Pitsch. A turbulent flame speed closure model for LES of industrial burner flows. Proceedings of the Summer Program, pages 169-179, 2000.

J. Grune, K. Sempert, H. Haberstroh, M. Kuznetsov, and T. Jordan. Experimental investigation of hydrogen-air deflagrations and detonations in semi-confined flat layers. Journal of Loss Prevention in the Process Industries, 26(2):317-323, oct 2011. ISSN 09504230. doi:10.1016/j.jlp.2011.09.008. URL http://linkinghub.elsevier.com/ retrieve/pii/S0950423011001677http: //dx.doi.org/10.1016/j.jlp.2011.09.008.

J Grune, K Sempert, A Friedrich, M Kuznetsov, and $\mathrm{T}$ Jordan. ScienceDirect Detonation wave propagation in semi-confined layers of hydrogen e air and hydrogen e oxygen mixtures. International Journal of Hydrogen Energy, 42(11):7589-7599, 2016. ISSN 0360-3199. doi:10.1016/j.ijhydene.2016.06.055. URL http://dx. doi.org/10.1016/j.ijhydene.2016.06.055.

Ryan W Houim and Robert T Fievisohn. The influence of acoustic impedance on gaseous layered detonations bounded by an inert gas. Combustion and Flame, 179:185-198, 2017. ISSN 0010-2180. doi:10.1016/j.combustflame.2017.02.001. URL http://dx.doi.org/10.1016/j. combustflame.2017.02.001.

H.I. Lee and D.S. Stewart. Calculation of linear detonation instability : one-dimensional instability of plane detonation. Journal of fluid mechanics, 216:103-132, 1990. ISSN 0022-1120. doi:10.1017/S0022112090000362.

J.H.S. Lee. The Detonation Phenomenon. Cambridge University Press, 2008. ISBN 0521897238. URL http://www. amazon.co. uk/Detonation-Phenomenon-John-H-Lee/ dp/0521897238http://www. amazon.fr/ The-Detonation-Phenomenon-ebook/dp/ B001EHE7RA.

W. Rudy, M. Kuznetsov, R. Porowski, a. Teodorczyk, J. Grune, and K. Sempert. Critical conditions of hydrogen-air detonation in partially confined geometry. Proceedings of the Combustion Institute, 34(2):1965-1972, jan 2013. ISSN 15407489. doi:10.1016/j.proci.2012.07.019. URL http: //linkinghub.elsevier.com/retrieve/ pii/S1540748912003112.

Wojciech Rudy, Mateusz Zbikowski, and Andrzej Teodorczyk. Detonations in hydrogen-methane-air mixtures in semi con fi ned fl at channels. 116:1479-1483, 2016. doi:10.1016/j.energy.2016.06.001.

W.P Sommers. The Interaction of a Detonation Wave with an Inert Boundary. $\mathrm{PhD}$ thesis, 1961.

K. Vaagsaether. Modelling of Gas Explosions. PhD thesis, Telemark University College - NTNU, Norway, 2010.

J. von Neumann. Theory of Detonation waves, John von Neumann: Collected Works. Pergamon Press, Oxford, 1963.

Ya.B. Zel'dovich. On the theory of the propagation of detonations on gaseous system. Journal of Experimental and Theoretical Physics, 10:542-568, 1940. 